\title{
BMJ Open Ultrasound-guided superficial cervical plexus block for analgesia in patients undergoing craniotomy via suboccipital retrosigmoid approach: study protocol of a randomised controlled trial
}

Kun Peng, Min Zeng, Jia Dong, Xiang Yan, Dexiang Wang, Shu Li (D) , Yuming Peng

To cite: Peng K, Zeng M, Dong J, et al. Ultrasoundguided superficial cervical plexus block for analgesia in patients undergoing craniotomy via suboccipital retrosigmoid approach: study protocol of a randomised controlled trial. BMJ Open 2020;10:e034003. doi:10.1136/ bmjopen-2019-034003

- Prepublication history for this paper is available online To view these files, please visit the journal online (http://dx.doi. org/10.1136/bmjopen-2019034003).

$\mathrm{KP}$ and $\mathrm{MZ}$ are joint first authors.

Received 01 September 2019 Revised 05 December 2019 Accepted 07 January 2020
Check for updates

(C) Author(s) (or their employer(s)) 2020. Re-use permitted under CC BY-NC. No commercial re-use. See rights and permissions. Published by BMJ.

Department of Anesthesiology, Beijing Tiantan Hospital, Capital Medical University, Beijing, China

Correspondence to Dr Yuming Peng;

florapym766@163.com

\section{ABSTRACT}

Introduction Scalp nerve block has been proven to be an alternative choice to opioids in multimodal analgesia. However, for the infratentorial space-occupying craniotomy, especially the suboccipital retrosigmoid craniotomy, scalp nerve block is insufficient.

Methods and analysis The study is a prospective, single-centre, randomised, paralleled-group controlled trial. Patients scheduled to receive elective suboccipital retrosigmoid craniotomy will be randomly assigned to the superficial cervical plexus block group or the control group. After anaesthesia induction, superficial cervical plexus nerve block will be performed under the guidance of ultrasound. The primary outcome is the cumulative consumption of sufentanil by the patient-controlled intravenous analgesia pump within 24 hours after surgery. Secondary outcomes include the cumulative consumption of sufentanil at other four time points and numerical rating scale pain severity score.

Ethics and dissemination The protocol (version number: 2.0, 10 April 2019) has been approved by the Ethics Review Committee of China Registered Clinical Trials (Ethics Review No. ChiECRCT-20190047). The findings of this study will be disseminated in peer-reviewed journals and at scientific conferences.

Trial registration number NCT04036812

\section{BACKGROUND}

The procedure of craniotomy was previously assumed to be less painful than other sites of surgeries. ${ }^{1-3}$ However, in the prospective study of patients undergoing craniotomy, Gottschalk et al found that the incidence of postoperative pain was as high as $87 \%$, among of $55 \%$ patients experienced moderate-tosevere pain. ${ }^{4}$

Post-craniotomy pain is mainly caused by scalp incision, with abundant free nerve endings. After incision, noxious stimulus signals from the scalp is received by the trigeminal branches or cervical plexus branches, and then transmitted through the
Strengths and limitations of this study

- This is the randomised controlled trial to observe the efficacy and safety of preoperative ultrasoundguided superficial cervical plexus block on postoperative analgesia in patients undergoing craniotomy via suboccipital retrosigmoid approach.

- The results will optimise postoperative analgesia in patients undergoing infratentorial craniotomy, thereby improving prognosis of the patients.

- This is a single-centre clinical trial design which might limit the generalisation of the conclusion.

trigeminal nucleus and the dorsal horn of the spinal cord to the hypothalamus and cerebral cortex. The whole process is regulated by a variety of inflammatory mediators, peripheral nerve pathways and central nervous system. ${ }^{5}$

The pain severity after craniotomy is closely associated with surgical approach. Gottschalk et al evaluated pain after craniotomy and found that the infratentorial approach was associated with severe postoperative pain and more perioperative analgesic requirements. ${ }^{4}$ Rimaaja et al reported that $32 \%$ of patients had no or only mild headache prior to removal of the cerebellopontine angle area mass, while $64 \%$ of patients developed severe headache after craniotomy. ${ }^{6}$ The high incidence of postoperative pain after craniotomy through infratentorial approach, especially suboccipital retrosigmoid approach may be related to the injury of neck muscles and posterior occipital muscles by the surgical approach, as well as the special position of the head and neck during craniotomy, leading to postoperative muscle spasm. ${ }^{78}$ Therefore, it is necessary to explore an ideal analgesic modality that can 
effectively provide surgical analgesia with minimal or no systemic changes for this population.

The skin incision is the main source of pain during craniotomy. Scalp nerve block has been proven to be an excellent alternative analgesic choice in supratentorial surgeries. ${ }^{9-11}$ However, for the suboccipital retrosigmoid craniotomy, the scalp innervation area is insufficient to cover the incision, resulting defective nerve blockage. Therefore, it is necessary to explore an analgesic modality to provide a more ideal analgesic regimen for patients undergoing suboccipital retrosigmoid craniotomy.

The cervical plexus is from the anterior branch of C1 to $\mathrm{C} 4$ cervical nerve, divided into superficial plexus and deep plexus. The cutaneous branches of the superficial cervical plexus include the lesser occipital nerve, the greater auricular nerve, transverse cervical nerve and supraclavicular nerve, innervating the incision area of the suboccipital sigmoid sinus approach craniotomy. ${ }^{12}$ Therefore, the superficial cervical plexus block (SCPB) is a potential candidate to satisfy the analgesic requirement of retrosigmoid craniotomy. Girard et al observed the effect of transitional analgesia from SCPB after elective infratentorial or occipital craniotomy in 30 patients. ${ }^{13}$ In the control group, $0.1 \mathrm{mg} / \mathrm{kg}$ morphine was administrated after close of the dura. It was found that the effect of SCPB on postoperative analgesia was not inferior to administration of morphine after dura closure. However, the sample size estimation they made is too small. Second, SCPB wasn't guided by ultrasound. The analgesic effect of
SCPB may not be fully guaranteed. In addition, no related adverse effects were reported in that study. However, the study provided us a feasible method of SCPB analgesia for suboccipital retrosigmoid craniotomy.

With the continual development of visualisation techniques, ultrasound-guided nerve blocks have become increasingly popular. ${ }^{14-16}$ Ultrasound-guided SCPB, the operator can directly see adjacent anatomical structure, inject the local anaesthetic into the correct anatomical level, avoid accidental injury during the puncture and avoid the unexpected deep cervical plexus block. Ultrasound-guided SCPB has the advantages of faster onset of action, less dosage, high success rate and fewer complications. ${ }^{1718}$

Therefore, we propose the hypothesis that preoperative ultrasound-guided SCPB could safely and effectively provide analgesia for patients undergoing craniotomy via suboccipital retrosigmoid approach. The objective is to compare the cumulative consumption of postoperative opioids between groups.

\section{METHODS}

\section{Study design}

This is a prospective, single-centre, randomised, paralleled-group controlled trial (figure 1.) being conducted at Beijing Tiantan Hospital, Capital Medical University, China. The study was registered within ClinicalTrials.gov on 29 June 2019. Preoperative interviews will

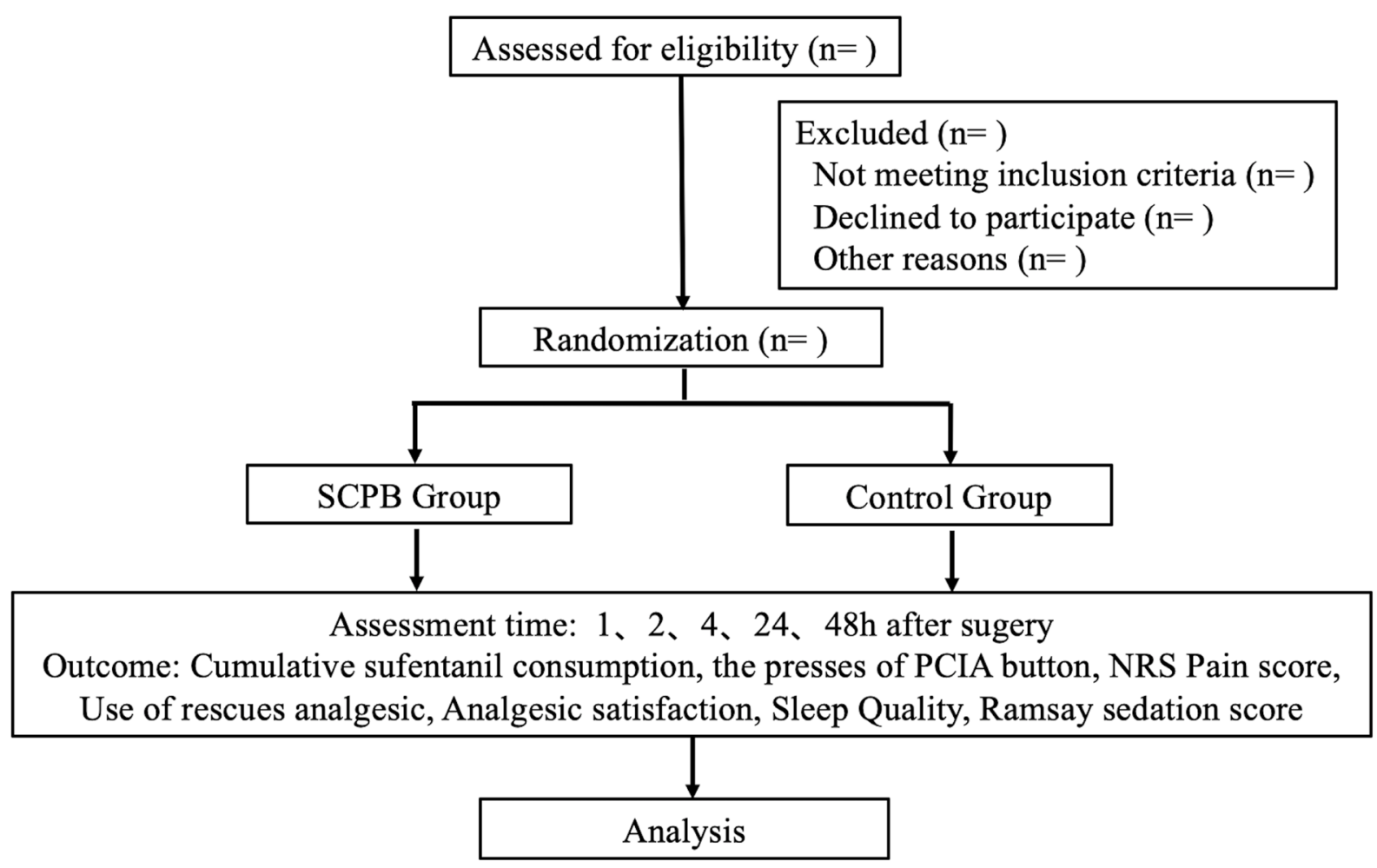

Figure 1 Consolidated Standards of Reporting Trials flow diagram. NRS, Numerical Rating Scale; PCIA, patientcontrolledintravenous analgesia; SCPB, superficial cervical plexus block. 


\begin{tabular}{|c|c|c|c|c|c|c|c|}
\hline \multicolumn{8}{|c|}{ STUDY PERIOD } \\
\hline \multirow{3}{*}{ TIMEPOINT } & Enrolment & \multirow{3}{*}{$\begin{array}{l}\text { Allocation } \\
\text { Sugery day }\end{array}$} & \multicolumn{5}{|c|}{ Post-allocation } \\
\hline & \multirow{2}{*}{-1day } & & \multicolumn{5}{|c|}{ Post-craniotomy(hour) } \\
\hline & & & 1 & 2 & 4 & 24 & 48 \\
\hline \multicolumn{8}{|c|}{ ENROLMENT } \\
\hline Eligibility screen & $x$ & & & & & & \\
\hline Informed consent & $x$ & & & & & & \\
\hline Allocation & & $x$ & & & & & \\
\hline \multicolumn{8}{|c|}{ INTERVENTIONS } \\
\hline SCPB group & & $x$ & & & & & \\
\hline Control group & & $x$ & & & & & \\
\hline \multicolumn{8}{|c|}{ ASSESSMENTS } \\
\hline Baseline variables & & $x$ & $x$ & $\mathrm{X}$ & $x$ & $x$ & $x$ \\
\hline Intraoperative data & & $x$ & & & & & \\
\hline Anesthesia recovery quality score & & & $x$ & $x$ & & & \\
\hline Ramsay Score & & & $x$ & $x$ & $x$ & $x$ & $x$ \\
\hline Cumulative sufentanil consumption of PCA & & & $x$ & $x$ & $x$ & $x$ & $x$ \\
\hline Requests of PCA & & & $x$ & $x$ & $x$ & $x$ & $x$ \\
\hline Dose and frequency of rescue analgesic & & & $x$ & $x$ & $x$ & $x$ & $x$ \\
\hline Pain intensity (NRS) & & & $x$ & $x$ & $x$ & $x$ & $x$ \\
\hline Analgesia Satisfaction & & & & & & $x$ & $x$ \\
\hline Sleep quality & & & & & & $x$ & $x$ \\
\hline Adverse Event & & & $x$ & $x$ & $x$ & $x$ & $x$ \\
\hline
\end{tabular}

Figure 2 Standard Protocol Items: Recommendations for Interventional Trials. NRS, Numerical Rating Scale; PCA, patientcontrolledanalgesia; SCPB, superficial cervical plexus block.

be conducted by specially trained research assistants to inform patients of the study objectives, risks and benefits and to obtain written informed consent from patients or legal representatives. The schedule of enrolment and assessments is shown in the Standard Protocol Items: Recommendations for Interventional Trials (figure 2).

\section{Study population}

Inclusion criteria

Patients scheduled to undergo elective suboccipital retrosigmoid craniotomy will be recruited for screening eligibility 1 day before surgery. Inclusion criteria include age between 18 and 65 years, and American Society of Anesthesiologists (ASA) physical status I to III.

\section{Exclusion criteria}

Exclusion criteria include refuse to provide written informed consent, local infection, preoperative impairment of consciousness and cognitive function, uncontrolled hypertension, inability to communicate; allergic to experimental drugs; history of drug abuse; history of chronic headache; aphasia and hearing impairment; second craniotomy; body mass index $<18.5 \mathrm{~kg} / \mathrm{m}^{2}$ or $>35.0 \mathrm{~kg} / \mathrm{m}^{2}$.

\section{Randomisation and blinding}

Randomisation will be conducted via a computergenerated table by an independent research assistant who will pack the allocation sequence with identical shape and size opaque envelopes and distribute to the researcher.
The researcher will open the envelopes and perform a SCPB or only puncture based on the grouping. Patients will be randomly assigned to two groups with a 1: 1 ratio. The researcher assistant, patients, the anaesthesiologist responsible for intraoperative management and outcome assessors will all be blinded to the allocation until the completion of the study analysis unless specific circumstances, such as the occurrence of a serious adverse event.

\section{Data collection}

After obtaining informed consent, an independent research assistant will initiate baseline information collection 1 day before surgery. Basic demographic information, including gender, age, vital signs, height, weight, past medical history/family history, medication history, supplementary examination, assessment (ASA classification, headache and severity, treatment, dizziness, tinnitus, facial paralysis, nausea, vomiting and other symptoms) will be collected. All personal information will be kept strictly confidential for research purposes only. The assessment of primary and other secondary outcomes will be performed by trained research assessors who are blinded to the group allocation.

\section{Intervention}

Patients will be randomly assigned to the SCPB group or the control group. Peripheral venous access will be established on arrival in operating room. After anaesthesia induction, SCPB will be performed under the guidance 
of ultrasound (Hitachi Company, Noblus) by the independent researcher who will not involve in intraoperative management or postoperative follow-up. Patients will be at supine position with ipsilateral shoulder relaxed and slightly elevated while head tilting to the opposite side. After marking the midpoint of the posterior border of clavicular head of the sternocleidomastoid muscle (about cricoid cartilage level, about 3 to $4 \mathrm{~cm}$ above the clavicle), an ultrasound probe (50 $\mathrm{mm}$ high frequency linear array) warped with sterilise plastic dress will be placed in the transverse position at the previous measuring mark. The scanning depth will be 3 to $4 \mathrm{~cm}$ and the focussing position will be 2 to $3 \mathrm{~cm}$. After confirming the sternocleidomastoid muscle, we move the probe backwards until the posterior border of the sternocleidomastoid muscle in the centre of the screen, and identify the investing fascia and prevertebral fascia from the shallow to the deep layer. Using long-axis in-plane technique, a $50 \mathrm{~mm}$ long, $20 \mathrm{G}$ short bevel needle will be inserted from the lateral border of sternocleidomastoid muscle. Under guidance of ultrasound, we will confirm the needle tip locating between the deep layer of investing fascia and the superficial layer of prevertebral fascia, close to the border of sternocleidomastoid muscle. After negative aspiration of blood and cerebrospinal fluid, $1 \mathrm{~mL}$ of $0.5 \%$ ropivacaine will be administered to confirm the location of the needle tip. Then, $10 \mathrm{~mL}$ of $0.5 \%$ ropivacaine will be infused on the superficial layer of prevertebral fascia. The puncture site will be covered with opaque infusion dressing after completing of SCPB.

In the control group, the puncture will also be performed by ultrasound guidance, covered with opaque infusion dressing but performed without infusion.

\section{Concomitant treatment}

Routine monitoring will include ECG, pulse oxygen saturation, non-invasive blood pressure (NIBP), body temperature, minimal alveolar concentration (MAC) of inhalation agent and bispectral index (BIS). Continuous arterial pressure, urine output and end-tidal carbon dioxide $\mathrm{PaO} 2\left(\mathrm{ETCO}_{2}\right)$ will be monitored after anaesthesia induction. All patients will be premedicated with midazolam $(0.05 \mathrm{mg} / \mathrm{kg})$ intravenously $5 \mathrm{~min}$ before anaesthesia induction. Anaesthesia will be induced with propofol (1.5 to $2.5 \mathrm{mg} / \mathrm{kg}$ ), sufentanil (0.3 to $0.4 \mu \mathrm{g}$ / $\mathrm{kg})$, and rocuronium $(0.9 \mathrm{mg} / \mathrm{kg})$ or cisatracurium $(0.2$ $\mathrm{mg} / \mathrm{kg}$ ). After tracheal intubation, mechanical ventilation will be performed, at a tidal volume of 6 to $8 \mathrm{~mL} / \mathrm{kg}$, a respiratory rate of 12 to $15 / \mathrm{min}$, an inspiratory/expiratory ratio of $1: 2$, a $50 \%$ fraction of inspired oxygen in the air and fresh gas at a flow rate of $2 \mathrm{~L} / \mathrm{min}$ to maintain the $\mathrm{ETCO}_{2}$ between 35 and $40 \mathrm{~mm} \mathrm{Hg}$.

Anaesthesia will be maintained with combined intravenous anaesthesia and inhalational anaesthesia. Along with the inhalational anaesthesia maintained with 0.5 MAC, infusion of remifentanil $(0.1$ to $0.4 \mu \mathrm{g} / \mathrm{kg} / \mathrm{min})$ and propofol (3 to $8 \mathrm{mg} / \mathrm{kg} / \mathrm{h}$ ) will be maintained to keep BIS values between 40 and 50. No muscle relaxant will be used during the procedure to meet intraoperative electronical physiological neuromonitoring requirements. No additional local anaesthetics or analgesics will be administered intraoperatively. Propofol and remifentanil infusion will be discontinued at the end of surgery.

The patients will be extubated after full recovery from anaesthesia and transferred to the post-anaesthesia care unit (PACU). The patients will remain in the PACU for 120 min and receive nasal oxygen inhalation with ECG, NIBP, pulse oximetry monitoring. Sufentanil-loaded electric analgesia pumps which preprogrammed by the research assistant will be connected to the patients for routine postoperative analgesia. The patient-controlled intravenous analgesia (PCIA) pumps will be filled with sufentanil $(100 \mu \mathrm{g})$ and ondansetron $(16 \mathrm{mg})$ diluted in $100 \mathrm{~mL}$ of $0.9 \%$ saline. This regimen will provide a bolus of $1 \mu \mathrm{g}$ sufentanil on demand with a 10 min lockout time, without continuous background infusion dose or loading dose. Insufficient postoperative analgesia will be defined as an Numerical Rating Scale (NRS) score $>4$ lasting over $15 \mathrm{~min}$ or $>6$. Once inadequate postoperative analgesia was confirmed, patients will receive rescue analgesic. If the patient vomit or report nausea for more than 15 min, rescue antiemetic will be administered. The type, the frequency and the dose of rescue analgesic and antiemetic will be recorded. The reason for administration will also be recorded giving drugs with analgesic or/and antiemetic.

Physiological parameters, the total doses of anaesthetic drugs and vasoactive drugs will be recorded. Fluid input and output will also be closely monitored and recorded. Anaesthesia and surgery duration will be summarised.

\section{Outcomes and safety measures}

The aim of this study is to observe the effect of SCPB on postoperative analgesia in patients with suboccipital retrosigmoid craniotomy. During preoperative visits, patients will be informed of the score how to assess the pain, analgesic satisfaction, sleep quality and anaesthesia recovery quality.

\section{Primary outcome}

The primary outcome is the cumulative consumption of sufentanil by the PCIA within 24 hours after surgery. The primary outcomes will be assessed by trained research assistants at 24 hours after surgery through reading the PCIA data.

\section{Secondary outcomes}

The secondary outcomes include the other efficacy parameters and safety outcomes.

1. The first time point that the patients use PCIA, the total and effective requests of PCIA at five different time points after surgery $(1,2,4,24,48$ hours $)$ and the cumulative consumption of sufentanil at four different time points (1, 2, 4, 48 hours) after surgery.

2. Pain Severity Score: Pain will be assessed at five time points after surgery. The degree of surgical incision 
pain will be assessed at rest and on movement NRS pain score. Insufficient postoperative analgesia is defined as an NRS score that exceeds 4 lasted for $15 \mathrm{~min}$ or exceeds 6 . Information of analgesic drugs administrated in case of insufficient postoperative analgesia was also recorded. Pain severity score in NRS is 0 to 10 , 0 representing no pain and 10 representing worst pain imaginable.

3. Anaesthesia Recovery Quality Score: Anaesthesia Steward Emergence Scale ${ }^{19}$ will be used at 1 and 2 hours after surgery to evaluate the recovery quality of anaesthesia. Anaesthesia recovery quality score will be assessed by the Anaesthesia Steward Emergence Scale which is divided into three parts: the degree of wakefulness $(2$ points for complete recovery, 1 point for response to stimulation, 0 point for no response to stimulation), the degree of airway patency ( 2 points for cough according to the doctor's order, 1 point for maintenance of airway patency without support, 0 point for support required for respiratory tract) and the degree of limb mobility ( 2 points for conscious activities of limbs, 1 point for unconscious activities of limbs, 0 point for no activities of limbs).

4. Analgesic satisfaction and sleep quality: Patient satisfaction with overall pain management and sleep quality will be evaluated separately at 24 and 48 hours after surgery using NRS. Analgesic satisfaction score in NRS is 0 to 10,0 representing extremely dissatisfied and 10 representing extremely satisfied. Sleep quality score in NRS is scored as 0 to 10,0 representing unable to sleep and 10 representing deep sleep.

5. Adverse events: Ramsay score ${ }^{20}$ and nausea and vomiting scores as well other adverse events (dizziness, fatigue, haematoma, local anaesthetic poisoning and hoarseness) will be evaluated at the five time points after surgery.

\section{Sample size calculation}

We estimate the sample size according to the primary outcome of postoperative-24-hour PCIA sufentanil consumption by using PASS 2011 software (NCSS LLC). Based on the previous literature, ${ }^{21}$ Akcil et aldemonstrated the mean $(95 \% \mathrm{CI})$ postoperative cumulative morphine consumption was $30 \mathrm{mg}$ (25 to 35 ) in the scalp block group and $50 \mathrm{mg}$ (40 to 60) in the control group. Considering that $1 \mathrm{mg}$ morphine is equivalent to $1 \mu \mathrm{g}$ sufentanil, we estimated the scalp block in their study reduced PCIA sufentanil consumption by $20 \mu \mathrm{g}$ within postoperative 24 hours. In the routine practice without SCPB, we also apply PCIA for the patients undergoing craniotomy via suboccipital retrosigmoid approach with the dosage of sufentanil as $50 \mu \mathrm{g}$ during the first 24 hours after surgery. So, we estimated the effect size of mean as $20 \mu \mathrm{g}$ with the SD of $30 \mu \mathrm{g}$ for the SCPB group comparing with the control group. The sample size of 106 patients will be sufficient to detect the difference at a two-tailed significant level of 0.05 and a power of $90 \%$ using Student's t-test, with a drop-out rate of $10 \%$.

\section{Statistical analysis}

Analysis will be done using SPSS software (V.23.0). We will apply the intention-to-treat and per-protocol analysis on the primary outcome. If necessary, consider the number of missing outcomes as poor prognosis and conduct sensitivity analysis. The continuous variables will be summarised with mean (SD) or median (IQR), depending on normality determined with Shapiro-Wilk test. Normally distributed and continuous variables will be compared with Student's t-test, while skewed variables will be compared using the Mann-Whitney $\mathrm{U}$ test. The categorical variables will be described as counts (percentages) and compared with $\mathrm{X}^{2}$ analysis or Fisher's exact test. The repeated measurement data will be analysed by repeated measurements of variance analysis. Bonferroni correction will be used for multiple comparisons. A significance level of $p<0.05$ was used to indicate statistical significance.

\section{Reporting of adverse events}

All adverse events will be closely monitored until a stable situation has been reached. The chief investigator will be informed of any serious adverse events and determine the severity and causality of these events. All adverse events associated with this study will be recorded and reported to the ethics committee as part of the annual report. The chief investigator will be responsible for getting the details about causes of adverse events, treatment measures, prognosis and reporting serious adverse events to the Ethics Committee immediately.

\section{Protocol amendment}

The chief investigator will be responsible for any decision to amend the protocol. If there is any modification (eg, changes to eligibility criteria, outcomes, analyses) the principle investigator will communicate and gain approval from the China Ethics Committee of Registering Clinical Trials prior to implementation, and communicate with relevant other parties (eg, investigators, trial participants, trial registries, journals, regulators)

\section{DISCUSSION}

An ideal analgesic should be able to provide analgesia for entire surgical period and with minimal or no systemic changes. Meanwhile, the interference on consciousness and postoperative neurological function should be minimised during the recovery and evaluation period. At present, PCIA with opioid is the most common analgesia modality for patients received craniotomy. ${ }^{21}{ }^{22}$ However, undesirable effects of opioids, including respiratory depression, nausea, vomiting, urinary retention, etc, not only bring discomfort to patients, but also affect neurological function evaluation by neurosurgeons. ${ }^{23}$ Besides opioids, non-steroidal anti-inflammatory drugs, another common analgesia agent is not suitable for postoperative analgesia after neurosurgery due to its effect on coagulation. ${ }^{24}$ Gabapentin is an adjuvant antiepileptic agent with some analgesic effects. Our previous research 
demonstrated that oral gabapentin relieved early postoperative pain, with increased depth of sedation in postcraniotomy, which indicated gabapentin was not the appropriate candidate for postoperative neurosurgical analgesia. ${ }^{25}$ Therefore, it is necessary to explore an ideal analgesic modality that can effectively provide surgical analgesia with minimal or no systemic changes for this population.

This is a prospective, single-centre, randomised, parallel-group controlled trial to assess the efficacy and safety of preoperative ultrasound-guided SCPB for analgesia in patients undergoing suboccipital retrosigmoid craniotomy. With the continuous development of ultrasound guidance technology, utilisation of visualised nerve block became more popular in clinical practice. ${ }^{15} 1618$ We design the current study to use ultrasound-guided SCPB to explore the efficacy and safety of postoperative analgesia in patients undergoing suboccipital sigmoid approach for craniotomy. Although ultrasound guidance greatly improves the efficacy and safety of the block, we still can't ignore the risk associated with the cervical plexus block. Therefore, in this study we will observe these complications such as haematoma, dizziness, fatigue, local anaesthetic poisoning and hoarseness. At the same time, in order to ensure the accuracy and consistency of ultrasound-guided puncture, the anaesthesiologists will receive specific training before the first patients are enrolled, the corresponding ultrasound image data of puncture will be preserved, so as to ensure the uniformity of block effect in each patient.

Although SCPB may reduce post-craniotomy pain, it is not routinely used in our current clinical practice. To maintain the analgesic effect on the patients in the control group, the patients will be given the same analgesic dosage regimen following the clinical routine of our medical centre, which fully ensure that the patients are safe and painless during surgery. Postoperative PCIA analgesia will be routinely given to all patients, and rescue analgesics will be promptly administered when analgesia is insufficient.

Our study will improve the ideal analgesic regimen for patients undergoing suboccipital retrosigmoid craniotomy, so as to reduce perioperative stress response and complications, improve patient satisfaction and early recovery.

\section{Patient and public involvement}

Patients and the public were not directly consulted in the development of the research question or outcome measures. Patients were not involved in the design, the recruitment and conduct of the study. At the completion of this trial, a manuscript will be prepared to present the trial results. Results of the final study will be disseminated to all study participants through their preferred method of communication indicated at the time of enrolment. The burden of intervention will not be taken by participants themselves.
Acknowledgements The authors would like to express their sincere gratitude to the colleagues of the Department of Anesthesiology and the Department of Neurosurgery, Beijing Tiantan Hospital, for their support and cooperation. The authors also would like to express their sincere gratitude to patient advisers for their cooperation in completing the trial.

Contributors KP, MZ, JD, DXW, XY, SL, YMP: conceived the study, contributed to the study design and analytical plans. MZ: drafted the protocol. All authors read and approved the final protocol.

Funding This work was supported by Beijing Municipal Administration of Hospitals Clinical Medical Development of Special Funding Support (grant number: ZYLX201708) and Ascent Plan (grant number: DFL20180502), Capital Development Research Fund Project (grant number: 2018-2-2044).

Competing interests None declared.

Patient consent for publication Not required.

Ethics approval This study is approved by the Ethics Review Committee of China Registered Clinical Trials (Ethics Review No. ChiECRCT-20190047).

Provenance and peer review Not commissioned; externally peer reviewed. Data availability statement Not applicable.

Open access This is an open access article distributed in accordance with the Creative Commons Attribution Non Commercial (CC BY-NC 4.0) license, which permits others to distribute, remix, adapt, build upon this work non-commercially, and license their derivative works on different terms, provided the original work is properly cited, appropriate credit is given, any changes made indicated, and the use is non-commercial. See: http://creativecommons.org/licenses/by-nc/4.0/.

\section{ORCID iDs}

Shu Li http://orcid.org/0000-0002-5625-067X

Yuming Peng http://orcid.org/0000-0002-2630-2467

\section{REFERENCES}

1 Dunbar PJ, Visco E, Lam AM. Craniotomy procedures are associated with less analgesic requirements than other surgical procedures. Anesth Analg 1999;88:335-40.

2 Maxwell LG, Buckley GM, Kudchadkar SR, et al. Pain management following major intracranial surgery in pediatric patients: a prospective cohort study in three academic children's hospitals. Paediatr Anaesth 2014;24:1132-40.

3 Molnár L, Simon Éva, Nemes R, et al. Postcraniotomy headache. J Anesth 2014;28:102-11.

4 Gottschalk A, Berkow LC, Stevens RD, et al. Prospective evaluation of pain and analgesic use following major elective intracranial surgery. J Neurosurg 2007;106:210-6.

5 Vadivelu N, Kai A, Tran D, et al. Options for perioperative pain management in neurosurgery. J Pain Res 2016;9:37-47.

6 Rimaaja T, Haanpää M, Blomstedt G, et al. Headaches after acoustic neuroma surgery. Cephalalgia 2007;27:1128-35.

7 de Gray LC, Matta BF. Acute and chronic pain following craniotomy: a review. Anaesthesia 2005;60:693-704.

8 Chowdhury T, Garg R, Sheshadri V, et al. Perioperative factors contributing the Post-Craniotomy pain: a synthesis of concepts. Front Med 2017;4:23.

9 Potters J-W, Klimek M. Local anesthetics for brain tumor resection: current perspectives. Local Reg Anesth 2018;11:1-8.

10 Chaki T, Sugino S, Janicki PK, et al. Efficacy and safety of a lidocaine and ropivacaine mixture for scalp nerve block and local infiltration anesthesia in patients undergoing awake craniotomy. J Neurosurg Anesthesiol 2016;28:1-5.

11 Hwang J-Y, Bang J-S, Oh C-W, et al. Effect of scalp blocks with levobupivacaine on recovery profiles after craniotomy for aneurysm clipping: a randomized, double-blind, and controlled study. World Neurosurg 2015;83:108-13.

12 Osborn I, Sebeo J. "Scalp block" during craniotomy: a classic technique revisited. J Neurosurg Anesthesiol 2010;22:187-94.

13 Girard F, Quentin C, Charbonneau S, et al. Superficial cervical plexus block for transitional analgesia in infratentorial and occipital craniotomy: a randomized trial. Can J Anaesth 2010;57:1065-70.

14 Re M, Blanco J, Gómez de Segura IA. Ultrasound-Guided nerve block anesthesia. Vet Clin North Am Food Anim Pract 2016;32:133-47.

15 Barrington MJ, Uda Y. Did ultrasound fulfill the promise of safety in regional anesthesia? Curr Opin Anaesthesiol 2018;31:649-55. 
16 Hoydonckx Y, Peng P. Echo-guided invasive pain therapy: indications and limitations. Curr Opin Anaesthesiol 2018;31:739-48.

17 Ho B, De Paoli M. Use of ultrasound-guided superficial cervical plexus block for pain management in the emergency department. $J$ Emerg Med 2018;55:87-95.

18 Senapathi TGA, Widnyana IMG, Aribawa IGNM, et al. UltrasoundGuided bilateral superficial cervical plexus block is more effective than landmark technique for reducing pain from thyroidectomy. $J$ Pain Res 2017;10:1619-22.

19 Steward DJ. A simplified scoring system for the post-operative recovery room. Can Anaesth Soc J 1975;22:111-3.

20 Porzio G, Aielli F, Verna L, et al. Efficacy and safety of deep, continuous palliative sedation at home: a retrospective, singleinstitution study. Support Care Cancer 2010;18:77-81.
21 Akcil EF, Dilmen OK, Vehid $\mathrm{H}$, et al. Which one is more effective for analgesia in infratentorial craniotomy? the scalp block or local anesthetic infiltration. Clin Neurol Neurosurg 2017;154:98-103.

22 Song J, Li L, Yu P, et al. Preemptive scalp infiltration with $0.5 \%$ ropivacaine and $1 \%$ lidocaine reduces postoperative pain after craniotomy. Acta Neurochir 2015;157:993-8.

23 Dunn LK, Naik BI, Nemergut EC, et al. Post-Craniotomy pain management: beyond opioids. Curr Neurol Neurosci Rep 2016;16:93.

24 Jian M, Li X, Wang A, et al. Flurbiprofen and hypertension but not hydroxyethyl starch are associated with post-craniotomy intracranial haematoma requiring surgery. Br J Anaesth 2014;113:832-9.

25 Zeng M, Dong J, Lin N, et al. Preoperative gabapentin administration improves acute postoperative analgesia in patients undergoing craniotomy. J Neurosurg Anesthesiol 2019;31:392-8. 